\title{
Detecting hidden defects from real data
}

\author{
by P.Bison ${ }^{1}$, F.Clarelli ${ }^{2}$ and G.Inglese ${ }^{2}$
}

${ }^{1}$ CNR-ITC, Padova, Italy. paolo.bison@itc.cnr.it

${ }^{2}$ CNR-IAC, Firenze, Italy.

\section{Abstract}

A defect $\sigma$ occurs on the inaccessible side of a metallic thin plate $\Omega$. We detect and evaluate $\sigma$ from real thermal data collected on the opposite side of $\Omega$.

Keywords Active InfraRed Thermography; Non-Destructive Evaluation; Partial Differential Equations; Domain Derivative; Fourier Transform.

AMS: $35 Q 80,45 Q 05,42 A 38$.

\section{Introduction}

The problem of corrosion detection and evaluation can be successfully faced by means of thermographic methods. In reflection technique a pulse of energy (flash light) is released on one surface of the solid to test while corrosion is present on the opposite (unaccessible) surface. Explicit formulae for the evaluation of material loss are available from literature [1, 2].

During the last twenty years, lock-in technique [3] (that applies a periodic heating source instead of the pulsed one) has been increasingly used in Thermal Non Destructive Testing aimed to defect detection. A typical drawback of lock-in is the long time data collection waiting for an average equilibrium of the temperature. The goal of the present paper is to obtain a reliable and unexpensive scheme of inversion, based on lock-in technique, to evaluate material loss of any shape and low percentages.

Let $C$ be the volumetric heat capacity $\left(C=c_{p} \rho\right.$ where $c_{p}$ is the specific heat and $\rho$ is the density) and $\lambda$ be the thermal conductivity of our specimen $\Omega_{0}$. The material is assumed homogeneous and isotropic so that $C, \rho$ and $\lambda$ are positive scalar constants in $(x, y$, $z)$. Let $u^{0}(x, y, z, t)$ be the temperature at the point $(x, y, z) \in \Omega_{0}$ at time $t$.

We suppose that $\Omega_{0}$ divides an outer aggressive environment from our laboratory. Let $S_{\text {Top }}=\{z=a\}$ be the inaccessible face of $\Omega_{0}$ in contact with the outside, while $S_{B o t}=\{z=0\}$ is the laboratory side. Also, we assume that the only effect of the external aggression to the specimen is the loss of an amount of matter, so that $S_{T o p}$ becomes an unflat surface, and a non-negative function $\sigma$ describes the deviation of damaged $S_{T o p}$ from the original plane i.e.

$$
\Omega_{\sigma}=\{(x, y, z): x, y \in(0,1) ; z=a-\sigma(x, y)\} .
$$

Let $u^{\sigma}(x, y, z, t)$ be the temperature at the point $(x, y, z) \in \Omega_{\sigma}$ at time $t$.

We apply a periodic heating source $\phi(t)$ on the surface $S_{b o t}^{\sigma}=\Omega_{\sigma}(x, y, z=0)$ and obtain a sequence of temperature maps $U_{k}^{\sigma}(x, y, 0)=u^{\sigma}\left(x, y, 0, t_{k}\right)$ from $\Omega_{\sigma}$. The sequence $U_{k}^{0}(x, y, 0)=u^{0}\left(x, y, 0, t_{k}\right)$ (background solution) is the thermal response of the undamaged specimen.

The inverse problem of active infrared thermography consists in detecting and evaluating the unknown function $\sigma$ once we know the heating term $\phi(t)$ and the measured thermal contrast $\delta U_{k}=U_{k}^{\sigma}-U_{k}^{0}$.

Our method is based on a sequence of mathematical steps. The main result of our mathematical analysis consist in a quasi-explicit discrete inversion formula. Moreover, thanks to our preprocessing step, data can be collected along two or three periods only when the system is still far from equilibrium. The scheme of our method is summarized in the following steps:

1. Domain derivative of the heat equation: The unknown $\sigma$ moves from the domain to the boundary condition at $z=a$.

2. Preprocessing of input data: the heating term $\phi(x ; y ; t)$ is $T$-periodic in time, while the contrast is $\delta U_{k}=t r e n d+F_{T}(x, y, t)+$ $R_{n}$, where $F_{T}$ is a $T$-periodic function and $R_{n}$ is a random noise. Denoising can be carried out by means of standard techniques. The trend term is subtracted so that we concentrate our effort on periodic data.

3. Fourier series expansion leads to a system of Helmoltz equations parametrized by the wave number. Since $\sigma$ is assumed to be time independent, we can select a single Helmoltz (elliptic) equation.

4. Integral formulation of this elliptic PDE leads to an infinite linear system of equations whose unknowns are the cosine-Fourier coefficients of $\sigma$. Some effort is required to build the infinite matrix of the 3-D system which turns out to be block diagonal made up of infinite Toeplitz-plus-Hankel submatrices. Finally, we obtain the following quasi explicit relation

$$
A_{m, n} \hat{\sigma}_{m, n}=\hat{W}_{m, n}, \quad m, n=0,1,2, \ldots
$$




\section{http://dx.doi.org/10.21611/qirt.2014.052}

We tested our method on a damaged carbon-steel sample sized $0.2(m) \times 0.15(m) \times 0.01(m)$

\section{The method}

The temperature distribution in $\Omega_{\sigma}$ fulfills the Boundary Value Problem (BVP) for the heat equation (2), (4), (3):

$$
\begin{gathered}
u_{t}=\frac{\lambda}{c_{p} \rho} \Delta u, \\
u(x, y, z, 0)=u^{e}, \\
\lambda u_{n}+h\left(u-u^{e}\right)-\phi=0, \quad \phi \neq 0 \text { only for } z=0 .
\end{gathered}
$$

The solution will be indicated by $u^{\sigma}$. In particular, $u^{0}$ is the background solution defined in $\Omega_{0}$. Condition (4) describes the energy exchange between the specimen and the environment. Here, $u_{n}$ is the outward normal derivative, $h$ is the surface heat exchange coefficient and $\phi=\phi_{0}\left(1+\sin \left(\frac{2 \pi}{T} t\right)\right)$ is the heat source applied. The coefficient $h$ is related to the geometry of the surface and to external environmental condition close to the boundary. In general $h_{t o p} \neq h_{\text {bot }}$. In our case, we observe that the value of $h_{t o p}$ changes in presence of damaging [5], but the deviation of $h$ from its background constant value can be considered negligible. The environmental temperature is $u^{e}$, the relevance of heat exchange between the specimen and the environment is actually related to the size of the Biot number $B=\frac{a h}{\lambda}$ and to the temperature change $u-u^{e}$.

The BVP defined by eqs. (2), (3) and (4) is well posed (see for example [6] [21, Chapter 2]).

\subsection{Domain derivative with respect to the perturbation}

The domain derivative was introduced in [7]. Since $|\sigma|$ is assumed small with respect to $a$, we write $\sigma=\epsilon \theta(\epsilon<<a$ and $0 \leq \theta(x, y) \leq 1$ for $(x ; y) \in(0,1) \times(0,1))$. Roughly speaking, the domain derivative $u^{\prime}$ of $u^{\sigma}$ with respect to the parameter $\epsilon$ for $\epsilon=0$ in the direction $\theta$, is the first order term of the expansion of $u$ in powers of $\epsilon$ :

$$
u^{\sigma}(x, y, z, t)=u^{0}(x, y, z, t)+\epsilon u^{\prime}(x, y, z, t)+o(\epsilon) .
$$

It comes from the definition of domain derivative that the scaled function $W=\epsilon u^{\prime}$ solves the boundary value problem (6)-(10)

$$
\begin{aligned}
W_{t} & =\alpha_{0} \Delta W \\
W_{x} & =W_{x}=0 \text { on }\{x=0\} \cup\{x=1\} \\
W_{y} & =W_{y}=0\{y=0\} \cup\{y=1\} \\
W_{z}-\gamma_{b o t} W & =0 \text { on }\left\{S_{B o t}\right\} \\
W_{z}+\gamma_{t o p} W & =\sigma\left(\frac{u_{t}^{0}}{\alpha_{0}}-\gamma_{\text {top }}^{2}\left(u^{0}-u_{t o p}\right)\right) \text { on }\left\{S_{t o p}\right\},
\end{aligned}
$$

where $\alpha_{0}=\frac{\lambda}{c_{p} \rho}, \gamma_{b o t}=h_{b o t} / \lambda, \gamma_{t o p}=h_{t o p} / \lambda$. The solution $W$ depends on the background solution $u^{0}$ so that it is implicitly related to the flux $\phi$. Moreover, we have

$$
W\left(x, y, 0, t_{k}\right)=u^{\sigma}\left(x, y, 0, t_{k}\right)-u^{0}\left(x, y, 0, t_{k}\right)+o\left(\epsilon^{2}\right) \approx \delta U_{k}(x, y)
$$

\subsection{Preprocessing: extraction of the periodic component}

We know that the functions $W$ and $u^{0}$ can be regarded as the sum of a time-periodic component (of period $T$ ) and a remainder $R$. It comes from the linearity of our boundary value problem, that the periodic components also fulfill (6)-(10) and (2), (4), (3) after minor straightforward changes. In order to not overload notation, in what follows we use the same notation $W$ and $u_{0}$ for the periodic components.

In practice, for all $(x, y)$ we identify a trend in $t$ of the function $\delta u(x, y, 0, t)$. We regard such a trend function of $(x, y, t)$ as the trace of the remainder $R$ on the face $z=0$ of the specimen. Once the trend is subtracted from the contrast, we get a $W(x, y, 0, t)$ approximatively periodic with period $T$. It will be useful in the next section.

\subsection{Helmoltz equations}

\subsubsection{Fourier transform of the periodic background}




\section{http://dx.doi.org/10.21611/qirt.2014.052}

Let $f_{k}$ be the $k$-th complex Fourier coefficient of a function $f$, i.e.

$$
\hat{f}_{k}(x, y, z)=\frac{1}{\tau} \int_{0}^{\tau} f(x, y, z, t) e^{-\frac{i 2 \pi k}{\tau} t} d t
$$

We apply Fourier transform to the periodic components of the background solution $u^{0}$ and $W$ : Fourier coefficients of $u^{0}$ fulfill the Helmoltz system (13)-(17)

$$
\begin{aligned}
\frac{2 \pi i k}{\tau}\left(1-\delta_{0, k}\right) \hat{u}_{k} & =\alpha_{0} \Delta \hat{u}_{k} ; \text { in } \Omega_{0} \\
\hat{u}_{k, x} & =0 ; \quad \text { on }\{x=0\} \cup\{x=1\} \\
\hat{u}_{k, y} & =0 ; \quad \text { on }\{y=0\} \cup\{y=1\} \\
\hat{u}_{k, n}+\gamma_{b}\left(-\hat{u}_{b} \delta_{0, k}-\frac{1}{h_{b}} \phi_{k}(x, y)\right) & =0 ; \quad \text { on }\left\{S_{\text {Bot }}\right\} \\
\hat{u}_{k, n}+\gamma_{t}\left(\hat{u}_{k}-\hat{u}_{t} \delta_{0, k}\right) & =0 ; \quad \text { on }\left\{S_{T o p}\right\},
\end{aligned}
$$

where $k \in \mathbf{Z}$,

$$
\delta_{0, k}=\left\{\begin{array}{l}
1, \text { if } k=0 \\
0, \text { otherwise }
\end{array}\right.
$$

and

$$
\hat{\phi}_{k}=\frac{1}{\tau} \int_{0}^{\tau} \phi(x, y, t) e^{-\frac{2 \pi i k t}{\tau}} d t= \begin{cases}\phi_{0} \frac{i}{2} & \text { if } k=-1 \\ \phi_{0} & \text { if } k=0 \\ -\phi_{0} \frac{i}{2} & \text { if } k=1 \\ 0 & \text { otherwise }\end{cases}
$$

Let $\hat{u}_{k}^{0}$ solve the above system (13)-(17) in $\Omega_{0}$. We can calculate it explicitly, by means of separation of variables. The solution for $k=0$ is eq. (19),

$$
\hat{u}_{0}^{0}(x, y, z)=\frac{1}{\gamma_{b}\left(1+\gamma_{t} a\right)+\gamma_{t}}\left[\gamma_{b} \gamma_{t}\left(\hat{u}_{t}-\hat{u}_{b}-\frac{\phi_{0}}{h_{b}}\right) z+\gamma_{b}\left(1+\gamma_{t} a\right) \hat{u}_{b}+\gamma_{t} \hat{u}_{t}+\gamma_{b}\left(1+\gamma_{t} a\right) \frac{\phi_{0}}{h_{b}}\right]
$$

and the solution for $k= \pm 1$ is

$$
\hat{u}_{k}^{0}(x, y, z)=-\frac{\hat{\phi}_{k}}{\lambda} \frac{\left(B_{k}-\gamma_{t}\right) e^{B_{k}(z-a)}+\left(B_{k}+\gamma_{t}\right) e^{-B_{k}(z-a)}}{\left(B_{k}-\gamma_{b}\right)\left(B_{k}-\gamma_{t}\right) e^{-B_{k} a}-\left(B_{k}+\gamma_{b}\right)\left(B_{k}+\gamma_{t}\right) e^{B_{k} a}},
$$

where

$$
B_{k}^{2}=\frac{2 \pi i k C_{0}}{\lambda \tau}
$$

\subsubsection{Fourier transform of the periodic domain derivative}

We apply Fourier transform to the periodic component of the domain derivative (6)-(10) obtaining the system

$$
\begin{aligned}
B_{k}^{2} \hat{W}_{k} & =\Delta \hat{W}_{k} ; \quad \text { in } \Omega_{0} \\
\hat{W}_{k, x} & =0 ; \quad \text { on }\{x=0\} \cup\{x=1\} \\
\hat{W}_{k, y} & =0 ; \quad \text { on }\{y=0\} \cup\{y=1\} \\
\hat{W}_{k, z}-\gamma_{b o t} \hat{W}_{k} & =0 ; \text { on }\left\{S_{B o t}\right\} \\
\hat{W}_{k, z}+\gamma_{\text {top }} \hat{W}_{k} & =\sigma u_{k}^{0}\left(B_{k}^{2}-\gamma_{\text {top }}^{2}\right) ; \quad \text { on }\left\{S_{T o p}\right\}
\end{aligned}
$$

At this point we can reformulate the inverse problem of Active Infrared Thermography as follows:

The lock-in version of inverse problem of active infrared thermography consists in detecting and evaluating the unknown function $\sigma$ once we know $u_{1}^{0}(x, y, a)$ (it depends on the first Fourier coefficient of $\left.\phi(t)\right)$ and the Fourier component for $k=1$ of the measured thermal contrast $\hat{W}_{1}(x, y, 0) \approx \epsilon \hat{\delta u_{1}}(x, y, 0)$.

Our goal is to write an analytical relation between $\sigma$ and the data starting from the integral equation of the weak solutions of the elliptic problem (22)-(26). Thereafter, we make use of some suitable orthogonal decomposition in the space of solutions.

\subsection{Final reconstruction}




\section{http://dx.doi.org/10.21611/qirt.2014.052}

From now on, for simplicity, we do not write explicitly the subscript index $k$. In the following, as in [4], we reduce our problem to an integral equation. From the Gauss-Green theorem, we have

$$
\int_{\partial \Omega_{0}} \frac{\partial v}{\partial n} \hat{W}=\int_{\partial \Omega_{0}} v \frac{\partial \hat{W}}{\partial n}
$$

where $\hat{W}$ is the solution of the system (22)-(26), and $v$ is a test function satisfying equations (31)-(35).

Now, we have for the right hand side of (27)

$$
\begin{aligned}
\int_{\partial \Omega_{0}} \frac{\partial v}{\partial n} \hat{W} & =\int_{S_{T o p}} \frac{\partial v}{\partial z} \hat{W}-\int_{S_{B o t}} \frac{\partial v}{\partial z} \hat{W}= \\
& =-\gamma_{t o p} \int_{S_{T o p}} v \hat{W}-\int_{S_{B o t}} \frac{\partial v}{\partial z} \hat{W}
\end{aligned}
$$

while the left hand side of (27) becomes

$$
\begin{aligned}
\int_{\partial \Omega_{0}} v \frac{\partial \hat{W}}{\partial n} & =\int_{S_{T o p}} v \frac{\partial \hat{W}}{\partial z}-\int_{S_{B o t}} v \frac{\partial \hat{W}}{\partial z}= \\
& =-\gamma_{t o p} \int_{S_{T o p}} v \hat{W}+\int_{S_{T o p}} v \sigma \hat{u}^{0}\left(B_{k}^{2}-\gamma_{t o p}\right)-\gamma_{b o t} \int_{S_{B o t}} v \hat{W} .
\end{aligned}
$$

Substituting eqs. (28) and (29) in the (27), we have

$$
\left(\frac{2 \pi i k}{\alpha \tau}-\gamma_{t}^{2}\right) \int_{S_{T o p}} v \sigma \hat{u}^{0}=-\int_{S_{B o t}}\left(v_{z}-\gamma_{b} v\right) \hat{W} .
$$

We choose a test function $v$ satisfying the system (31)-(35).

$$
\begin{aligned}
\frac{2 \pi i k}{\alpha \tau} v & =\Delta v ; \quad \text { in } \Omega_{0} \\
v_{x} & =0 ; \quad \text { on }\{x=0\} \cup\{x=1\} \\
v_{y} & =0 ; \quad \text { on }\{y=0\} \cup\{y=1\} \\
v_{z}-\gamma_{b} v & =\cos \left(\frac{\pi m}{L_{x}} x\right) \cos \left(\frac{\pi n}{L_{y}} y\right) ; \quad \text { on } S_{B o t} \\
v_{z}+\gamma_{t} v & =0 ; \quad \text { on } S_{T o p}
\end{aligned}
$$

Solving the system (31)-(35) by separation of variables, we obtain:

1. for $k=0$, the solutions (36) and (37),

$$
\begin{gathered}
v_{0,0}(x, y, z)=\frac{1}{\gamma_{b}+\gamma_{t}+\gamma_{b} \gamma_{t} a}\left(\gamma_{t} z-\left(1+\gamma_{t} a\right)\right) \\
v_{m, n}(x, y, z)=\cos \left(\frac{\pi m}{L_{x}} x\right) \cos \left(\frac{\pi n}{L_{y}} y\right) f_{m, n}^{0}(z), \quad m, n=0,1,2, \ldots
\end{gathered}
$$

where

$$
f_{m, n}^{0}(z)=\frac{\left[\left(\eta_{m, n}-\gamma_{t}\right) e^{\eta_{m, n}(z-a)}+\left(\eta_{m, n}+\gamma_{t}\right) e^{-\eta_{m, n}(z-a)}\right]}{\left(\eta_{m, n}-\gamma_{b}\right)\left(\eta_{m, n}-\gamma_{t}\right) e^{-\eta_{m, n} a}-\left(\eta_{m, n}+\gamma_{b}\right)\left(\eta_{m, n}+\gamma_{t}\right) e^{\eta_{m, n} a}},
$$

and $\eta_{m, n}=\sqrt{\left(\frac{\pi m}{L_{x}}\right)^{2}+\left(\frac{\pi n}{L_{y}}\right)^{2}}$.

2. for $k= \pm 1$ we obtain the solution (39)

$$
v_{m, n}^{k}(x, y, z)=\cos \left(\frac{\pi m}{L_{x}} x\right) \cos \left(\frac{\pi n}{L_{y}} y\right) f_{m, n}^{1}(z), \quad m, n=0,1,2, \ldots
$$

where

$$
f_{m, n}^{1}(z)=\frac{\left[\left(\zeta_{m, n}^{k}+\gamma_{t}\right) e^{\zeta_{m, n}^{k}(a-z)}+\left(\zeta_{m, n}^{k}-\gamma_{t}\right) e^{-\zeta_{m, n}^{k}(a-z)}\right]}{\left(\zeta_{m, n}^{k}-\gamma_{b}\right)\left(\zeta_{m, n}^{k}-\gamma_{t}\right) e^{-\zeta_{m, n}^{k} a}-\left(\zeta_{m, n}^{k}+\gamma_{b}\right)\left(\zeta_{m, n}^{k}+\gamma_{t}\right) e^{\zeta_{m, n}^{k} a}},
$$

and $\zeta_{m, n}^{k}=\sqrt{B_{k}^{2}+\left(\frac{\pi m}{L_{x}}\right)^{2}+\left(\frac{\pi n}{L_{y}}\right)^{2}}, \quad m, n=0,1,2, \ldots$ 


\section{http://dx.doi.org/10.21611/qirt.2014.052}

Let us assume that

$$
b_{1}^{k}=\left(\frac{2 \pi i k}{\alpha \tau}-\gamma_{t}^{2}\right)
$$

and we develop in Fourier cosine series $\sigma(41)$ and $\hat{W}(42)$;

$$
\begin{gathered}
\sigma=\sum_{j, q} \sigma_{j, q} \cos \left(j \frac{\pi}{L_{x}} x\right) \cos \left(q \frac{\pi}{L_{y}} y\right), \\
\hat{W}=\sum_{j, q} \hat{W}_{j, q} \cos \left(j \frac{\pi}{L_{x}} x\right) \cos \left(q \frac{\pi}{L_{y}} y\right) .
\end{gathered}
$$

Finally, for $k=1$, we find out

$$
\sigma_{m, n}=-\frac{\hat{W}_{m, n}}{b_{1}^{1} \hat{u}^{0}(a) f_{m, n}^{1}(a)}, \forall m, n
$$

\section{Real data experiments}

Case 1. Carbon-Steel layer, with the properties listed in table 1.

Table 1: Carbon-Steel properties.

\begin{tabular}{|c|c|c|}
\hline Property & Value & Dim. \\
\hline$\rho_{s}$ & 7850 & $\mathrm{Kg} / \mathrm{m}^{3}$ \\
$c_{s}$ & 470 & $\mathrm{~J} /\left(\mathrm{Kg}^{\circ} \mathrm{K}\right)$ \\
$\lambda$ & 52 & $\mathrm{~W} /\left(\mathrm{m}^{\circ} \mathrm{K}\right)$ \\
\hline
\end{tabular}

\section{References}

[1] V. Vavilov, E. Grinzato, P.G. Bison, S. Marinetti, M. Bales, Inversion for hidden corrosion characterization: theory and applications, Int. J. Heat Mass Trans. 39 (1996) 355-371.

[2] E. Grinzato, V. Vavilov, P.G. Bison, S. Marinetti, Hidden corrosion detection in thick metallic components by transient IR thermography, Infrared Physics \& Technology 49 (2007) 234-238.

[3] G. Busse, D. Wu and W. Karpen, Thermal wave imaging with phase sensitive modulated thermography, Journal of Applied Physics, 71(8), 3962-3965 (1992).

[4] Fasino D., Inglese G. and Mariani F., "Corrosion detection in conductiong boundaries: II. Linearization, stability and discretization". Inverse Problems, 23, pp. 1101-1114, 2007.

[5] Inglese G., “An inverse problem in corrosion detection”. Inverse Problems, 13, no. 4, pp. 977-994, 1997.

[6] Salsa S., "Partial Differential Equation in Action". Springer-Verlag Italia, Milan, 2008.

[7] Simon J., "Differentiation with respect to the domain in boundary value problems". Numer. Funct. Anal. Optim., 2, pp. 649-687, 1980. 